\title{
QUANTUM PRINCIPAL BUNDLES AND THEIR CHARACTERISTIC CLASSES
}

\author{
MIĆO ĐURĐEVIĆ \\ Instituto de Matematicas, UNAM, Area de la Investigacion Cientifica \\ Circuito Exterior, Ciudad Universitaria \\ México DF, CP 04510, Mexico \\ E-mail: miko@servidor.unam.mx
}

\begin{abstract}
A general theory of characteristic classes of quantum principal bundles is presented, incorporating basic ideas of classical Weil theory into the conceptual framework of noncommutative differential geometry. A purely cohomological interpretation of the Weil homomorphism is given, together with a geometrical interpretation via quantum invariant polynomials. A natural spectral sequence is described. Some interesting quantum phenomena appearing in the formalism are discussed.
\end{abstract}

1. Introduction. Classical theory of characteristic classes is one of the most powerful theoretical tools in the study of the internal topological structure of the fibered spaces. In this paper we are going to present the basic structural elements of a noncommutativegeometric [2,3] generalization of classical Weil theory [8] of characteristic classes for principal bundles. All considerations are logically based on a general theory of quantum principal bundles, developed in $[4,5]$. This paper does not contain the proofs. A detailed exposition of the theory of quantum characteristic classes can be found in $[6]$.

General constructions of characteristic classes are presented in Section 3. We shall consider two levels of generality. The first one is given by quantum principal bundles equipped with a differential calculus which admits certain special connections, satisfying a property called regularity. It turns out that in this case the classical Weil construction of characteristic classes can be directly incorporated [5] into the quantum context. The domain of the quantum Weil homomorphism is consisting of the adjointly-invariant elements of the braided-symmetric algebra built over the left-invariant part of the given bicovariant *-calculus over the structure quantum group. These elements are quantum counterparts of the standard invariant polynomials on the structure group Lie algebra. The second level of generality is given by the arbitrary quantum principal bundles, and

1991 Mathematics Subject Classification: Primary 55R65, 53C05, 16W30; Secondary 57R20. 
the arbitrary calculi on them. To handle this, we shall present a different construction of characteristic classes, in the framework of which a natural cohomological interpretation of the domain of the Weil homomorphism is given. In both cases the Weil homomorphism takes the values from the cohomology algebra of the differential calculus over the base space (or its graded center, in the regular case). Section 4 is devoted to the study of a natural spectral sequence, which can be associated to an arbitrary quantum principal bundle.

In the next section basic elements of the differential calculus over quantum principal bundles and the theory of connections are collected. The last section contains concluding examples. A particular attention is given to the purely quantum phenomena appearing in the formalism.

2. Geometrical background. Let us consider a compact matrix [9] quantum group $G$, represented by a Hopf *-algebra $\mathcal{A}$, interpreted as consisting of polynomial functions on $G$. Let $\phi, \epsilon$ and $\kappa$ be the coproduct, counit and the antipode map.

Let $M$ be a quantum space, represented by a ${ }^{*}$-algebra $\mathcal{V}$. Let $P=(\mathcal{B}, i, F)$ be a quantum principal $G$-bundle over $M$. Here $\mathcal{B}$ is a ${ }^{*}$-algebra representing $P$ as a quantum space, while $i: \mathcal{V} \rightarrow \mathcal{B}$ and $F: \mathcal{B} \rightarrow \mathcal{B} \otimes \mathcal{A}$ are unital *-homomorphisms playing the role of the dualized projection of $P$ on $M$ and the right action of $G$ respectively. The map $F$ is coassociative and counital, while $i$ is injective and its image coincides with the $F$-fixed point subalgebra of $\mathcal{B}$.

Let us assume that the complete differential calculus on $G$ is described by [4] the universal envelope $\Gamma^{\wedge}$ of a given first-order [10] bicovariant ${ }^{*}$-calculus $\Gamma$. This is a bicovariant graded-differential *-algebra given by $\Gamma^{\wedge}=\Gamma^{\otimes} / S^{\wedge}$, where $\Gamma^{\otimes}$ is the tensor bundle algebra over $\Gamma$ and $S^{\wedge}$ is a quadratic ideal. Let $\Gamma_{i n v}$ be the left-invariant part of $\Gamma$, and similarly let us denote by $\Gamma_{i n v}^{\wedge, \otimes}$ and $S_{i n v}^{\wedge}$ the left-invariant parts of the corresponding spaces. Let us mention that $\Gamma_{i n v}^{\otimes}$ is just the standard tensor algebra over $\Gamma_{i n v}$. We have $\Gamma_{i n v}^{\wedge}=\Gamma_{i n v}^{\otimes} / S_{i n v}^{\wedge}$, and the ideal $S_{i n v}^{\wedge}$ is generated by

$$
S_{\text {inv }}^{\wedge 2}=\left\{\pi\left(a^{(1)}\right) \otimes \pi\left(a^{(2)}\right) \mid a \in \mathcal{R}\right\},
$$

where $\mathcal{R} \subseteq \operatorname{ker}(\epsilon)$ is the right $\mathcal{A}$-ideal which corresponds to $\Gamma$, and $\pi: \mathcal{A} \rightarrow \Gamma_{\text {inv }}$ is the canonical projection map.

Let $\Omega(P)$ be a graded-differential *-algebra representing the calculus on $P$. By definition [5], this means that $\Omega^{0}(P)=\mathcal{B}$, and that $\mathcal{B}$ generates the differential algebra $\Omega(P)$. Furthermore, we require that the right action $F$ is extendible to a graded-differential homomorphism $\widehat{F}: \Omega(P) \rightarrow \Omega(P) \widehat{\otimes} \Gamma^{\wedge}$. This extension is unique and hermitian. Furthermore

$$
(\widehat{F} \otimes \mathrm{id}) \widehat{F}=(\mathrm{id} \otimes \widehat{\phi}) \widehat{F}
$$

where $\widehat{\phi}: \Gamma^{\wedge} \rightarrow \Gamma^{\wedge} \widehat{\otimes} \Gamma^{\wedge}$ is the unique (hermitian and grade-preserving) differential extension of the coproduct map.

The formula $F^{\wedge}=\left(\right.$ id $\left.\otimes p_{*}\right) \widehat{F}$, where $p_{*}: \Gamma^{\wedge} \rightarrow \mathcal{A}$ is the projection map, defines the right action $F^{\wedge}: \Omega(P) \rightarrow \Omega(P) \otimes \mathcal{A}$ of $G$ on $\Omega(P)$. 
Let $\omega: \Gamma_{i n v} \rightarrow \Omega(P)$ be a connection on $P$. This means that $\omega$ is a hermitian intertwiner between the adjoint action $\varpi: \Gamma_{i n v} \rightarrow \Gamma_{i n v} \otimes \mathcal{A}$ and $F^{\wedge}$, such that

$$
\pi_{v} \omega(\vartheta)=1 \otimes \vartheta
$$

where $\pi_{v}: \Omega(P) \rightarrow \mathfrak{v e r}(P)$ is the verticalization homomorphism, and $\mathfrak{v e r}(P)$ is the gradeddifferential *-algebra representing 'verticalized' differential forms on the bundle. We can write $\mathfrak{v e r}(P)=\mathcal{B} \otimes \Gamma_{i n v}^{\wedge}$, at the level of graded vector spaces. The set of all connections on $P$ is non-empty. It is naturally a real affine space. The above mentioned adjoint action is explicitly given by

$$
\varpi \pi=(\pi \otimes \mathrm{id}) \operatorname{ad} \quad \operatorname{ad}(a)=a^{(2)} \otimes \kappa\left(a^{(1)}\right) a^{(3)} .
$$

Equivalently, we can say that connections on $P$ are hermitian first-order linear maps $\omega: \Gamma_{i n v} \rightarrow \Omega(P)$ satisfying the identity

$$
\widehat{F} \omega(\vartheta)=\sum_{k} \omega\left(\vartheta_{k}\right) \otimes c_{k}+1 \otimes \vartheta
$$

where $\varpi(\vartheta)=\sum_{k} \vartheta_{k} \otimes c_{k}$.

The elements of a graded $*_{\text {-subalgebra }}$

$$
\mathfrak{h o r}(P)=\widehat{F}^{-1}\{\Omega(P) \otimes \mathcal{A}\},
$$

are naturally interpretable as horizontal forms on $P$. The above algebra is $F^{\wedge}$-invariant. In the framework of this interpretation, it is natural to define differential forms on $M$ as $F^{\wedge}$-invariant elements of $\mathfrak{h o r}(P)$. These elements form a graded-differential ${ }^{*}$-subalgebra $\Omega(M)$ of $\Omega(P)$. Equivalently, $\Omega(M)$ is the $\widehat{F}$-fixed-point subalgebra of $\Omega(P)$.

We say that $\omega$ is regular iff

$$
\omega(\vartheta) \varphi=(-1)^{\partial \varphi} \sum_{k} \varphi_{k} \omega\left(\vartheta \circ c_{k}\right)
$$

for each $\varphi \in \mathfrak{h o r}(P)$, where $\sum_{k} \varphi_{k} \otimes c_{k}=F^{\wedge}(\varphi)$ and $\circ$ denotes the canonical right $\mathcal{A}$ module structure given by ()$\circ a=\kappa\left(a^{(1)}\right)() a^{(2)}$. Let us observe that regular connections graded-commute with the elements of $\Omega(M)$. We say that $\omega$ is multiplicative iff it is extendible to a unital homomorphism $\omega^{\wedge}: \Gamma_{i n v}^{\wedge} \rightarrow \Omega(P)$. This extension is unique, and hermitian.

It turns out that regular connections are all multiplicative or not at the same time. If the calculus on the bundle is such that regular connections are not multiplicative then it is possible to 'renormalize' it, by factorizing $\Omega(P)$ through an appropriate gradeddifferential *-ideal, which measures a lack of multiplicativity of regular connections. Such a factorization does not change the first-order calculus. In terms of the renormalized calculus, regular connections are multiplicative. In what follows it will be assumed that regularity implies multiplicativity.

The above definition of the map $\omega^{\wedge}$ can be extended to non-multiplicative connections, with the help of the formula

$$
\omega^{\wedge}=\omega^{\otimes} \iota .
$$


Here $\iota: \Gamma_{i n v}^{\wedge} \rightarrow \Gamma_{i n v}^{\otimes}$ is a fixed grade-preserving hermitian section of the factorization map, which intertwines the adjoint actions of $G$, while $\omega^{\otimes}: \Gamma_{i n v}^{\otimes} \rightarrow \Omega(P)$ is the unital multiplicative extension of $\omega$.

The formulas

$$
\begin{aligned}
(\psi \otimes \eta)(\varphi \otimes \vartheta) & =(-1)^{\partial \eta \partial \varphi} \sum_{k} \psi \varphi_{k} \otimes\left(\eta \circ c_{k}\right) \vartheta \\
(\varphi \otimes \vartheta)^{*} & =\sum_{k} \varphi_{k}^{*} \otimes\left(\vartheta^{*} \circ c_{k}^{*}\right)
\end{aligned}
$$

introduce a graded *-algebra structure in the graded vector space

$$
\mathfrak{v h}(P)=\mathfrak{h o r}(P) \otimes \Gamma_{i n v}^{\wedge} .
$$

Let $m_{\omega}: \mathfrak{v h}(P) \rightarrow \Omega(P)$ be a linear map defined by

$$
m_{\omega}(\varphi \otimes \vartheta)=\varphi \omega^{\wedge}(\vartheta) .
$$

It turns out that $m_{\omega}$ is bijective. It intertwines the corresponding actions of $G$. Moreover, if $\omega$ is regular and multiplicative then $m_{\omega}$ is a *-algebra isomorphism.

The quantum counterpart of the horizontal projection operator can be defined by

$$
h_{\omega}=\left(\mathrm{id} \otimes p_{*}\right) m_{\omega}^{-1} .
$$

It projects $\Omega(P)$ onto $\mathfrak{h o r}(P)$. Following classical differential geometry, the covariant derivative operator is naturally defined by

$$
D_{\omega}=h_{\omega} d
$$

Finally, the curvature operator $R_{\omega}: \Gamma_{i n v} \rightarrow \Omega(P)$ can be defined as the composition

$$
R_{\omega}=D_{\omega} \omega
$$

The identity

$$
R_{\omega}=d \omega-\langle\omega, \omega\rangle
$$

is the counterpart of the classical structure equation. Here \langle\rangle are the brackets naturally associated to the embedded differential $\delta: \Gamma_{i n v} \rightarrow \Gamma_{i n v} \otimes \Gamma_{i n v}$, given by composing $d$ and the embedding $\iota$. Let us observe that the curvature operator $R_{\omega}$ implicitly depends on the $\operatorname{map} \delta$.

Let $\mathrm{I}\left[\Gamma_{i n v}^{\otimes}\right] \subseteq \Gamma_{i n v}^{\otimes}$ be the graded *-subalgebra consisting of elements invariant under the adjoint action $\varpi: \Gamma_{i n v}^{\otimes} \rightarrow \Gamma_{i n v}^{\otimes} \otimes \mathcal{A}$. The formula

$$
W^{\omega}(\vartheta)=R_{\omega}^{\otimes}(\vartheta)
$$

defines a ${ }^{*}$-homomorphism $W^{\omega}: \mathrm{I}\left[\Gamma_{i n v}^{\otimes}\right] \rightarrow \Omega(M)$. Here $R_{\omega}^{\otimes}: \Gamma_{i n v}^{\otimes} \rightarrow \Omega(P)$ is the corresponding unital multiplicative extension.

We shall denote by $Z(M)$ the graded centre of $\Omega(M)$. It is a graded-differential * subalgebra of $\Omega(M)$.

If $\omega$ is regular then the following identity holds

$$
R_{\omega}(\vartheta) \varphi=\sum_{k} \varphi_{k} R_{\omega}\left(\vartheta \circ c_{k}\right),
$$


for each $\varphi \in \mathfrak{h o r}(P)$. In particular, the curvature $R_{\omega}$ commutes with all elements of $\Omega(M)$, and hence the image of $W^{\omega}$ is contained in $Z(M)$. The covariant derivative of regular connections is always hermitian, and satisfies the graded Leibniz rule.

\section{Quantum characteristic classes}

3.1. The regular case. Let us assume that the bundle admits regular (and multiplicative) connections. A large class of examples of such bundles is given by non-commutative frame structures [7]. In this special case there exists a canonical regular connection $\omega$, corresponding to the Levi-Civita connection in classical geometry.

Let us assume that $\omega$ is a regular connection on a quantum principal bundle $P$ with the calculus $\Omega(P)$. Applying essentially the same transformations as in the classical case [8], it follows that $W^{\omega}(\vartheta)$ is closed, for each $\vartheta \in \mathrm{I}\left[\Gamma_{i n v}^{\otimes}\right]$.

LEMMA 1. The cohomological class of $W^{\omega}(\vartheta)$ in $Z(M)$ is independent of the choice of a regular connection $\omega$.

In other words, there exists the intrinsic unital *-homomorphism

$$
W: \mathrm{I}\left[\Gamma_{i n v}^{\otimes}\right] \rightarrow H Z(M) \quad W(\vartheta)=\left[W^{\omega}(\vartheta)\right],
$$

where $H Z(M)$ is the corresponding cohomology algebra. This map is a quantum counterpart of the Weil homomorphism.

Let $\sigma: \Gamma_{i n v}^{\otimes 2} \rightarrow \Gamma_{i n v}^{\otimes 2}$ be the canonical [10] braid operator. It is explicitly given by

$$
\sigma(\eta \otimes \vartheta)=\sum_{k} \vartheta_{k} \otimes\left(\eta \circ c_{k}\right)
$$

where $\varpi(\vartheta)=\sum_{k} \vartheta_{k} \otimes c_{k}$.

It turns out that the quantum Weil homomorphism is further factorizable through the ideal $\mathcal{J}$ generated by the space $\operatorname{im}(I-\sigma) \subseteq \Gamma_{\text {inv }}^{\otimes 2}$. This follows from the commutation identity

$$
R_{\omega}(\eta) R_{\omega}(\vartheta)=\sum_{k} R_{\omega}\left(\vartheta_{k}\right) R_{\omega}\left(\eta \circ c_{k}\right)
$$

The elements of the factoralgebra $\Sigma=\Gamma_{\text {inv }}^{\otimes} / \mathcal{J}$ are quantum counterparts of the polynoms over the Lie algebra of the structure group. The adjoint action $\varpi$ is naturally projectable to $\varpi_{\Sigma}: \Sigma \rightarrow \Sigma \otimes \mathcal{A}$. Let us denote by $\mathrm{I}[\Sigma] \subseteq \Sigma$ the subalgebra of elements invariant under $\varpi_{\Sigma}$. We have $\mathrm{I}[\Sigma]=\mathrm{I}\left[\Gamma_{\text {inv }}^{\otimes}\right] /\left(\mathrm{I}\left[\Gamma_{\text {inv }}^{\otimes}\right] \cap \mathcal{J}\right)$, and from the commutation relations defining $\Sigma$ it follows that $\mathrm{I}[\Sigma]$ is a central subalgebra of $\Sigma$.

In summary, we have constructed the intrinsic map

$$
W: \mathrm{I}[\Sigma] \rightarrow H Z(M)
$$

In classical differential geometry $G$ is a classical Lie group, and $\Gamma$ is the standard calculus. Furthermore $\Gamma_{i n v}$ is the dual space of the corresponding Lie algebra, $\sigma$ is the standard transposition and $\mathrm{I}[\Sigma]$ is the algebra of the standard invariant polynomials. The base space is a classical smooth manifold, equipped with the classical differential calculus.

3.2. The general case. In generalizing the theory of characteristic classes to the level of arbitrary bundles, we shall follow the idea of universality. Algebraic expressions gener- 
ating characteristic classes should be the same for all bundles. The following observation is the starting point.

Proposition 2. Let us assume that $\omega$ is a regular and multiplicative connection on $P$. Then the image of the map $W^{\omega}$ consists of differential forms on $M$ which are expressible in terms of $\omega$ and $d \omega$.

Let $\Omega$ be a differential algebra generated by the first-order space $\Gamma_{i n v}$ with the only relation $d(1)=0$. The *-involution on $\Gamma_{i n v}$ naturally extends to $\Omega$, so that $d: \Omega \rightarrow \Omega$ is a hermitian map. The cohomology of $\Omega$ is trivial-we have $H(\Omega)=\mathbb{C}$.

There exists the unique graded-differential homomorphism $\widetilde{\varpi}: \Omega \rightarrow \Omega \widehat{\otimes} \Gamma^{\wedge}$ such that

$$
\widetilde{\varpi}(\vartheta)=\varpi(\vartheta)+1 \otimes \vartheta
$$

for each $\vartheta \in \Gamma_{i n v}$. Furthermore this map is hermitian and

$$
(\widetilde{\varpi} \otimes \mathrm{id}) \widetilde{\varpi}=(\mathrm{id} \otimes \widehat{\phi}) \widetilde{\varpi} .
$$

It is also possible to introduce a natural right action $\varpi_{\wedge}: \Omega \rightarrow \Omega \otimes \mathcal{A}$, extending the adjoint action map $\varpi$. Explicitly,

$$
\varpi_{\wedge}=\left(\mathrm{id} \otimes p_{*}\right) \widetilde{\varpi}
$$

Let $\neg \subseteq \Omega$ be the $\widetilde{\varpi}$-fixed-point graded differential *-subalgebra.

Let $P=(\mathcal{B}, i, F)$ be a quantum principal $G$-bundle over $M$, endowed with a calculus $\Omega(P)$. Let $\omega$ be an arbitrary connection on $P$.

Proposition 3. (i) There exists the unique homomorphism $\widehat{\omega}: \Omega \rightarrow \Omega(P)$ of differential algebras extending $\omega$. This map is hermitian. Moreover, it intertwines $\widetilde{\omega}$ and $\widehat{F}$. In particular, it follows that $\widehat{\omega}(T) \subseteq \Omega(M)$.

(ii) The induced cohomology map $W: H(ד) \rightarrow H(M)$ is independent of the choice of a connection $\omega$.

The constructed map $W$ is a counerpart of the Weil homomorphism, at the level of general quantum principal bundles. Characteristic classes are therefore labeled by the elements of $H(\top)$.

The algebra $\Omega$ possesses various properties characteristic to differential algebras describing the calculus on quantum principal bundles. In particular, it is possible to introduce a natural decomposition

$$
\Omega \leftrightarrow \mathfrak{h}(\Omega) \otimes \Gamma_{i n v}^{\wedge}=\mathfrak{v h}(\Omega) \quad \varphi \iota(\vartheta) \leftrightarrow \varphi \otimes \vartheta
$$

where $\mathfrak{h}(\Omega) \subseteq \Omega$ is a graded $*$-subalgebra describing 'horizontal elements', defined by

$$
\mathfrak{h}(\Omega)=\widetilde{\varpi}^{-1}(\Omega \otimes \mathcal{A}) \text {. }
$$

It follows that $\varpi_{\wedge}[\mathfrak{h}(\Omega)] \subseteq \mathfrak{h}(\Omega) \otimes \mathcal{A}$, in other words $\mathfrak{h}(\Omega)$ is $\varpi_{\wedge}$-invariant. The algebra $\neg$ is the $\varpi_{\wedge}$-fixed point subalgebra of $\mathfrak{h}(\Omega)$.

The first step in computing characteristic classes is to find the cocycles of 7 . The following is a prescription of constructing these cocycles. Every cocycle $w \in 7^{k}$ with $k \geq 1$ is of the form $w=d \varphi$, where $\varphi$ is some $\varpi_{\wedge}$-invariant element of $\Omega^{k-1}$. Then we have the equivalence

$$
w \in\urcorner \Longleftrightarrow d\{\widetilde{\varpi}(\varphi)-\varphi \otimes 1\}=0 .
$$


LEMmA 4. Let $\mathcal{C} \subseteq \Omega \otimes \Gamma^{\wedge}$ be the subcomplex spanned by elements of the form $c=$ $\widetilde{\varpi}(\varphi)-\varphi \otimes 1$, where $\varphi \in \Omega$ is $\varpi_{\wedge}$-invariant. Then the following natural correspondence holds

$$
\left.H^{n}(\urcorner\right) \leftrightarrow H^{n-1}(\mathcal{C})
$$

In particular, for every quantum principal bundle $P$ the cocycles representing characteristic classes are exact, as classes on the bundle, with $\widehat{\omega}(w)=d \widehat{\omega}(\varphi)$. Therefore, the above introduced elements $\varphi$ play the role of universal Chern-Simons forms.

Another possible approach in computing quantum characteristic classes consists in finding explicitly horizontal forms, and computing invariant elements ( $\Leftrightarrow$ the algebra 7 ). The algebra $\mathfrak{h}(\Omega)$ is invariant under the action of the operator

$$
\ell(\vartheta, \varphi)=\vartheta \varphi-(-1)^{\partial \varphi} \sum_{k} \varphi_{k}\left(\vartheta \circ c_{k}\right)
$$

where $\vartheta \in \Gamma_{i n v}$ and $\sum_{k} \varphi_{k} \otimes c_{k}=\varpi_{\wedge}(\varphi)$.

Proposition 5. The algebra $\mathfrak{h}(\Omega)$ is the minimal $\ell$-invariant subalgebra of $\Omega$ containing the elements $R(\vartheta)=d \vartheta-\delta(\vartheta)$ and the elements from $S_{i n v}^{\wedge 2}$.

Let us now return to the structures admitting regular and multiplicative connections. If we work only with regular connections, then the cohomological construction of the Weil homomorphism should be appropriately refined, by factorizing $\Omega$ through the ideal which takes into account the regularity property.

Let $\mathcal{K} \subseteq \Omega$ be the ideal generated by elements from $S_{i n v}^{\wedge 2}$, and the elements of the form

$$
\begin{aligned}
& j_{3}(\eta, \vartheta)=\ell(\eta, R(\vartheta))=\eta R(\vartheta)-\sum_{k} R\left(\vartheta_{k}\right)\left(\eta \circ c_{k}\right) \\
& j_{4}(\eta, \vartheta)=R(\eta) R(\vartheta)-\sum_{k} R\left(\vartheta_{k}\right) R\left(\eta \circ c_{k}\right),
\end{aligned}
$$

where $\eta, \vartheta \in \Gamma_{i n v}$ and $\varpi(\vartheta)=\sum_{k} \vartheta_{k} \otimes c_{k}$.

It follows that $\Gamma_{i n v}^{\wedge}$ is a subalgebra of $\Omega_{*}=\Omega / \mathcal{K}$, in a natural manner. We have

$$
\widetilde{\varpi} R(\vartheta)=\sum_{k} R\left(\vartheta_{k}\right) \otimes c_{k} \quad R(\vartheta)^{*}=R\left(\vartheta^{*}\right) .
$$

The ideal $\mathcal{K}$ is invariant under the actions of $\widetilde{\varpi}, *$ and $d$.

The maps $\widetilde{\varpi}, d, *, \varpi_{\wedge}$ are hence projectable to $\Omega_{*}$. Obviously, projected maps (that will be denoted by the same symbols) are in the same algebraic relations as the original ones. Let us introduce the horizontal part $\mathfrak{h}\left(\Omega_{*}\right)=\widetilde{\varpi}^{-1}\left(\Omega_{*} \otimes \mathcal{A}\right)$ of $\Omega_{*}$, which is a $\varpi_{\wedge}$-invariant ${ }^{*}$-subalgebra of $\Omega_{*}$. Applying a similar reasoning as in [5] it follows that

Lemma 6. (i) The product map in $\Omega_{*}$ induces a graded vector space isomorphism

$$
\mathfrak{h}\left(\Omega_{*}\right) \otimes \Gamma_{i n v}^{\wedge} \leftrightarrow \Omega_{*} .
$$

(ii) The map $R: \Gamma_{i n v} \rightarrow \Omega_{*}$ can be uniquely extended to a unital *-homomorphism $R: \Sigma \rightarrow \Omega_{*}$. The extended $R$ maps isomorphically $\Sigma$ onto $\mathfrak{h}\left(\Omega_{*}\right)$. Moreover, $R$ intertwines $\varpi_{\Sigma}$ and $\varpi_{\wedge}$. 
Let us formulate a regular counterpart of Proposition 3. Let us consider a quantum principal $G$-bundle $P=(\mathcal{B}, i, F)$ over $M$, with a calculus $\Omega(P)$ admitting regular and multiplicative connections. Let $\omega$ be an arbitrary regular connection. Let is denote by $\beth \subseteq \Omega_{*}$ the $\widetilde{\varpi}$-fixed point subalgebra of $\Omega_{*}$.

Proposition 7. ( $i)$ There exists the unique homomorphism $\widehat{\omega}: \Omega_{*} \rightarrow \Omega(P)$ of differential algebras extending the connection $\omega$. The map $\widehat{\omega}$ is hermitian, and

$$
(\widehat{\omega} \otimes \mathrm{id}) \widetilde{\varpi}=\widehat{F} \widehat{\omega} \text {. }
$$

In particular, $\widehat{\omega}(\beth) \subseteq \Omega(M)$.

(ii) The following identities hold

$$
\widehat{\omega} R=R_{\omega} \quad \widehat{\omega} D=D_{\omega} \widehat{\omega}
$$

where $D: \Omega_{*} \rightarrow \Omega_{*}$ is a first-order antiderivation specified by

$$
D R(\vartheta)=0 \quad D \vartheta=R(\vartheta) .
$$

(iii) The induced cohomology map $W: H(\beth) \rightarrow H(M)$ is independent of the choice of a connection $\omega$.

(iv) We have $H(\beth)=\beth=\mathrm{I}[\Sigma]$, in a natural manner. This gives a connection with the definition of the Weil homomorphism given in the previous subsection.

The above introduced map $D$ is called the universal covariant derivative. We have $D^{2}=0$.

Let $d_{v h}: \Omega_{*} \rightarrow \Omega_{*}$ be 'the universal' vertical differential. By definition, this map is acting in the following way

$$
d_{\imath h}(\varphi \otimes \vartheta)=\sum_{k} \varphi_{k} \otimes \pi\left(c_{k}\right) \vartheta+\varphi \otimes d(\vartheta)
$$

where $\varpi_{\wedge}(\varphi)=\sum_{k} \varphi_{k} \otimes c_{k}$.

LEMMA 8. The following identities hold

$$
\begin{aligned}
D d_{\imath h}+d_{\imath h} D & =0 \\
D+d_{\imath h} & =d .
\end{aligned}
$$

Let us observe that the map $D$ acts skew-diagonally, with respect to a natural bigrading in $\Omega_{*}$. This implies that the cohomology algebra $H_{D}\left(\Omega_{*}\right)$ is naturally bigraded, too.

4. The spectral sequence. Let us consider a quantum principal bundle $P$ endowed with a differential structure $\Omega(P)$.

For each $k \geq 0$ let $\Omega_{k}(P) \subseteq \Omega(P)$ be the space consisting of elements having the 'vertical order' less or equal $k$. In other words,

$$
\Omega_{k}(P)=\widehat{F}^{-1}\left(\Omega(P) \otimes \Gamma_{k}^{\wedge}\right)
$$


where $\Gamma_{k}^{\wedge}$ consists of forms having degrees not exceeding $k$. These spaces form a filtration of $\Omega(P)$, and the following compatibility properties hold:

$$
\Omega_{k}(P)^{*}=\Omega_{k}(P) \quad d \Omega_{k}(P) \subseteq \Omega_{k+1}(P) \quad \Omega_{k}(P)=\sum_{j \geq 0}^{\oplus} \Omega_{k}^{j}(P) .
$$

Let us consider a graded-differential *-algebra

$$
\amalg(P)=\sum_{k \geq 0}^{\oplus} \Omega_{k}(P),
$$

where the grading is given by numbers $k$, and the differential *-structure is induced from $\Omega(P)$. Let $\risingdotseq: \amalg(P) \rightarrow \amalg(P)$ be the first-order map induced by the inclusions $\Omega_{k}(P) \subseteq$ $\Omega_{k+1}(P)$. By definition, this is a monomorphism of differential *-algebras. Let $\mathfrak{g r}(P)$ be the graded-differential *-algebra associated to the introduced filtration. In other words, we have a short exact sequence

$$
0 \longrightarrow \amalg(P) \stackrel{\triangleright}{\longrightarrow} \amalg(P) \longrightarrow \mathfrak{g r}(P) \longrightarrow 0
$$

of differential *-algebras.

The space $\Omega_{k}(P)$ is linearly spanned by elements of the form

$$
w=\varphi \omega\left(\vartheta_{1}\right) \ldots \omega\left(\vartheta_{j}\right)
$$

where $\varphi \in \mathfrak{h o r}(P)$ and $j \leq k$, while $\omega$ is an arbitrary connection. The algebra $\mathfrak{g r}(P)$ is invariantly isomorphic to the algebra $\mathfrak{v} h(P)$ of 'vertically-horizontally' decomposed forms. Explicitly, the isomorphism $\mathfrak{v h}(P) \leftrightarrow \mathfrak{g r}(P)$ is given by

$$
\left(\varphi \otimes\left(\vartheta_{1} \ldots \vartheta_{k}\right)\right) \leftrightarrow\left[\varphi \omega\left(\vartheta_{1}\right) \ldots \omega\left(\vartheta_{k}\right)+\Omega_{k-1}(P)\right] .
$$

We shall assume that the two algebras are identified, with the help of the above isomorphism. We shall also assume that the calculus $\Gamma$ is such that only scalar elements of $\mathcal{A}$ are anihilated by the differential map.

The factor-differential $d_{v h}: \mathfrak{v h}(P) \rightarrow \mathfrak{v h}(P)$ is given by

$$
d_{v h}(\varphi \otimes \vartheta)=(-1)^{\partial \varphi} \sum_{k} \varphi_{k} \otimes \pi\left(c_{k}\right) \vartheta+(-1)^{\partial \varphi} \varphi \otimes d(\vartheta)
$$

where $\sum_{k} \varphi_{k} \otimes c_{k}=F^{\wedge}(\varphi)$.

It turns out that

$$
H[\mathfrak{v} \mathfrak{h}(P)]=\Omega(M) \widehat{\otimes} H\left(\Gamma_{i n v}^{\wedge}\right),
$$

in a natural manner.

Let $E(P)=\left\{E_{r}(P) \mid r \in \mathbb{N}\right\}$ be the spectral sequence associated to the introduced short exact sequence. The introduced filtration of $\Omega(P)$ induces a filtration of the ${ }_{-}$ algebra $H(P)$ of cohomology classes. We have

$$
H_{k}(P)=\sum_{j}^{\oplus} H_{k}^{j}(P) .
$$

Applying general theory [1], it follows that the introduced spectral sequence is convergent, and that $E_{\infty}(P)$ coincides with the graded *-algebra associated to the filtered $H(P)$. 
By construction $E_{1}(P)=H[\mathfrak{v} \mathfrak{h}(P)]$. Furthermore, it turns out that the differential $d_{1}$ is given by

In particular,

$$
d_{1}(w \otimes[\vartheta])=d w \otimes[\vartheta]
$$

$$
E_{2}(P)=H(M) \widehat{\otimes} H\left(\Gamma_{i n v}^{\wedge}\right) .
$$

A similar considerations can be applied to algebras $\Omega$ and $\Omega_{*}$, in particular we can associate natural spectral sequences to these algebras. The spectral sequence $E(\Omega)$ converges to the trivial cohomology $H(\Omega)=\mathbb{C}$. Therefore the spaces $H^{k}(ד)$ are naturally filtered. On the other hand, the cohomology of $\Omega_{*}$ is generally non-trivial. It measures, in a certain sense, the irregularity of the braiding $\sigma$.

The trivial convergence information is insufficient to compute the cohomology algebra $H(7)$. However, in various interesting special cases the spectral sequence degenerates (as in classical geometry), and the triviality property is sufficient to determine all cohomology classes.

5. Concluding remarks. We have assumed that the higher-order calculus on $G$ is described by the universal envelope $\Gamma^{\wedge}$. This corresponds to the maximal solution. All the constructions with differential forms on quantum principal bundles can be performed dealing with the bicovariant $\sigma$-exterior [10] algebra $\Gamma^{\vee}$, instead of $\Gamma^{\wedge}$. This corresponds to the minimal appropriate higher-order calculus, the essential property is that the coproduct map is extendible to a homomorphism $\phi^{\vee}: \Gamma^{\vee} \rightarrow \Gamma^{\vee} \widehat{\otimes} \Gamma^{\vee}$ of graded-differential *-algebras.

Moreover, all the constructions can be performed also for 'intermediate' higher-order calculi, described by higher-order graded-differential ${ }^{*}$-ideals $S_{\star} \subseteq \Gamma^{\wedge}$ satisfying

$$
\widehat{\phi}\left(S_{\star}\right) \subseteq S_{\star} \widehat{\otimes} \Gamma^{\wedge}+\Gamma^{\wedge} \widehat{\otimes} S_{\star} .
$$

Changing the higher-order calculus over $G$ directly influences the algebra of higherorder horizontal forms, and in particular the higher-order part of $\Omega(M)$.

The cohomological formulations of the two levels of the theory (regular and general) are essentially the same. However, it is interesting to observe that the corresponding universal characteristic classes radically differ, if the calculus on the group is sufficiently 'irregular'.

For example, at the level of general bundles there exist generally nontrivial classes in odd dimensions, in contrast to the regular case where all classes are expressible in terms of the curvature map. Another important difference between two levels is concerning the existence of the Chern-Simons forms. At the general level, all characteristic classes vanish, as cohomology classes of the bundle. In contrast to this, generally there exist regular characteristic classes, nontrivial as classes on the bundle. This is a manifestation of the already mentioned non-triviality of the cohomology of $\Omega_{*}$. In particular, such classes have no analogs at the level of general differential structures. On the other hand, under certain regularity assumptions on the calculus over the structure group, universal classes for the regular and the general case coincide. This essentially simplifies the work with bundles without regular connections. 
The higher-order calculus on $G$ can be always maximally adopted, from the point of view of the appearance of 3-dimensional characteristic classes. Let us consider this point in more details.

In dimension 2, Chern-Simons forms are labeled by closed elements of $\Gamma_{i n v}^{\wedge 2}$, invariant under the actions of $\varpi$ and $\sigma$. Let $S_{3} \subseteq \Gamma^{\wedge}$ be the ideal generated by the elements of the form $d(\psi)$, where $\psi \in \Gamma_{i n v}^{\wedge 2}$ satisfy

$$
\sigma(\psi)=\psi \quad \varpi(\psi)=\psi \otimes 1 .
$$

Lemma 9. The space $S_{3}$ is a graded-differential ${ }^{*}$-ideal in $\Gamma^{\wedge}$. Moreover,

$$
\widehat{\phi}\left(S_{3}\right) \subseteq S_{3} \widehat{\otimes} \Gamma^{\wedge}+\Gamma^{\wedge} \widehat{\otimes} S_{3} .
$$

Let us pass to the factor-calculus $\Gamma^{\triangle}=\Gamma^{\wedge} / S_{3}$, in the framework of which the elements $\psi$ are closed. It follows that

LEMMA 10. Relative to the factorized calculus

$H^{3}(ד)=\left\{\right.$ Special elements $\left.\psi \in \Gamma_{i n v}^{\wedge 2}\right\} / d\left\{\varpi\right.$-invariant elements of $\left.\Gamma_{i n v}\right\}$.

\section{References}

[1] R. Bott \& W. Tu, Differential Forms in Algebraic Topology, Springer-Verlag New-York (1982).

[2] A. Connes, Non-commutative differential geometry, Extrait des Publications Mathématiques, IHES 62 (1986).

[3] A. Connes, Noncommutative Geometry, Academic Press (1994).

[4] M. Đurđević, Geometry of Quantum Principal Bundles I, Commun Math Phys 175 (3) 457-521 (1996).

[5] M. Đurđević, Geometry of Quantum Principal Bundles II-Extended Version, Preprint, Instituto de Matematicas, UNAM, México (1994).

[6] M. Đurđević, Characteristic Classes of Quantum Principal Bundles, Preprint, Instituto de Matematicas, UNAM, México (1995).

[7] M. Đurđević, General Frame Structures on Quantum Principal Bundles, Preprint, Instituto de Matematicas, UNAM, México (1995).

[8] S. Kobayashi \& K. Nomizu, Foundations of Differential Geometry, Interscience Publishers New York, London (1963).

[9] S. L. Woronowicz, Compact Matrix Pseudogroups, Commun Math Phys $111613-665$ (1987).

[10] S. L. Woronowicz, Differential Calculus on Compact Matrix Pseudogroups/ Quantum Groups, Commun Math Phys 122 125-170 (1989). 